\title{
Multi-Parameter Quantum Groups Related to Link-Diagrams *
}

\author{
Paolo Cotta-Ramusino ${ }^{1 \star \star}$ and Maurizio Rinaldi ${ }^{2}$ \\ ${ }^{1}$ Department of Mathematics, University of North Carolina, Chapel Hill, NC, USA, and I.N.F.N. \\ Sezione di Milano, Milano, Italy \\ 2 Physics Department, Harvard University, Cambridge, MA 02138, USA
}

Received March 29, 1991

\begin{abstract}
We apply the Faddeev-Reshetikhin-Taktajan method for the construction of Quantum Groups to the Yang-Baxter matrices which are related to the invariants of oriented links in $\Sigma \times[0,1]$, where $\Sigma$ is a non-trivial 2-dimensional surface. We obtain multi-parameter ribbon Hopf algebras that differ in many respects from their one-parameter counterparts. Among the main differences we mention the existence of a non-central quantum determinant and the fact that the number of independent generators is higher than in the one-parameter case.
\end{abstract}

\section{Introduction}

It has been pointed out that invariants of links in the 3-dimensional space $\Sigma \times[0,1]$, where $\Sigma$ is a (open or closed) 2-dimensional surface, can be conveniently described by an algebraic structure defined on the set of oriented link-diagrams on $\Sigma([1,2])$.

In order to define such an algebraic structure and construct the invariants, we associate to each edge of a given link-diagram an integer (label) in $\{1, \ldots, N\}$; some properties being required for this labelling procedure (including a conservation (Kirchhoff) law for the labels of the four edges meeting at any given vertex). Furthermore we associate to each vertex a given function of the indeterminate variables $x, x^{-1}, z, z^{-1}$ depending on whether $v$ is of type $L_{+}$or $L_{-}$(here we use the standard terminology of knot theory) and depending on the labels of the four edges meeting at $v$. In this way we are able to construct a partition function which defines the link-invariants [3].

\footnotetext{
* Supported in part by the Italian National Institute for Nuclear Physics (I.N.F.N.) and by the National Science Foundation (N.S.F.) under Grant DMS 89-01975 and Grant DMS/PHY $88-16214$

$\star \star$ Visiting Fulbright Scholar, on leave of absence from the University of Trento, Italy
} 
We can represent collectively the above rules, by encoding them into an invertible matrix $R \in \operatorname{End}\left(\mathbf{C}^{N} \otimes C^{N}\right)$ whose entries $R_{i, j}^{k, l}$ are defined as follows. Given any four edges of the diagram, meeting at a vertex $v$ of type $L_{+}$, we associate the four indices $i, j, k, l$ to the possible value of the labels of the lower incoming edge (i), of the upper incoming edge $(j)$, of the lower outgoing edge $(k)$, of the upper outgoing edge $(l)$ and we set $R_{i, j}^{k, l}$ equal to the following functions of $x$ and $z: x^{2}$ when $i=j=k=l ; z^{2}$ when $i=k<j=l ; z^{-2}$ when $i=k>j=l ; x^{2}-x^{-2}$ when $i=l<j=k$ and zero otherwise.

If we consider to the contrary the case when the vertex $v$ is of type $L_{-}$and if we keep exactly the same correspondences defined above between labels of the four edges and indices of the relevant matrix, then the labelling rules are encoded in a matrix which is the inverse of $R$.

We can in fact consider a more refined version of the above rules, by replacing the variables $z$ and $z^{-1}$ with the set of variables $z_{k, l}$ subjected to the condition $z_{l, k}=z_{k, l}^{-1}$. In this case we have to set $R_{i, j}^{k, l}=z_{k, l}^{2}$ for $i=k \neq j=l$, the other entries being equal to the ones previously defined.

The possibility of constructing link-invariants via the above labelling procedure is related to some specific properties of the matrix $R$, including the fact that it satisfies the Yang-Baxter equation.

It is then natural to try to describe the quantum groups which are connected to these Yang-Baxter matrices. This is the problem which will be addressed in this paper.

Since we want to describe a quantum group starting from a Yang-Baxter matrix, the obvious approach will be to consider the method of Faddeev-ReshetikhinTaktajan for the construction of quantum groups [4].

Let us now give a short account of this method. Let $R \in \operatorname{End}\left(\mathbf{C}^{N} \otimes \mathbf{C}^{N}\right)$ be a generic invertible Yang-Baxter matrix, namely an invertible matrix satisfying the following relation for operators in $\operatorname{End}\left(\mathbf{C}^{N} \otimes \mathbf{C}^{N} \otimes \mathbf{C}^{N}\right)$ :

$$
R_{1,2} R_{1,3} R_{2,3}=R_{2,3} R_{1,3} R_{1,2} .
$$

Here we use the following standard notation:

$$
R_{m, n}: \mathbf{C}^{N} \otimes \cdots \otimes \mathbf{C}^{N} \rightarrow \mathbf{C}^{N} \otimes \cdots \otimes \mathbf{C}^{N},
$$

which is, by definition, the operator given by the matrix $R$ acting on the $m^{\text {th }}$ and the $n^{\text {th }}$ factor and by the identity operator acting on the remaining factors.

We define the algebra $A$ as the free associative algebra over the complex numbers generated by the $N^{2}$ elements $t_{l}^{k}(k, l=1, \ldots, N)$ (represented collectively by the matrix $T$ ) and the unit 1 .

$A$ is a bialgebra with comultiplication:

$$
\Delta\left(t_{l}^{k}\right) \equiv \sum_{j} t_{j}^{k} \otimes_{C} t_{l}^{j} ; \quad \Delta(1)=1 \otimes 1,
$$

and counit: $\eta(1)=1, \eta\left(t_{l}^{k}\right)=\delta_{l}^{k}$.

The bialgebra $A_{R}$ is defined as the quotient of $A$ with respect to bilateral ideal generated by

$$
\sum_{i, j} R_{i, j}^{k, l} t_{p}^{i} t_{m}^{j}-\sum_{i, j} t_{j}^{l} t_{i}^{k} R_{p, m}^{i, j}
$$



Here the indices are defined as follows: $R\left(e_{k} \otimes e_{l}\right) \equiv \sum_{i, j} R_{k, l}^{i, j}\left(e_{i} \otimes e_{j}\right)$, for a given basis
$\left\{e_{s}\right\}$ in $\mathbf{C}^{N}$.

Let $M_{N}(\mathbf{C})$ denote the algebra of $N \times N$ complex matrices. We consider now the bialgebra $U_{R} \equiv \operatorname{Hom}\left(A_{R}, \mathbf{C}\right)$ (dual to $\left.A_{R}\right)$ and its sub-bialgebra $U_{R}^{1}$ generated by the entries of the following four algebra homomorphisms ${ }^{1}$ :

$$
\Lambda^{ \pm}: A_{R} \rightarrow M_{N}(\mathbf{C}), \quad \Xi^{ \pm}: A_{R} \rightarrow M_{N}(\mathbf{C})^{\text {opp }},
$$

which are defined as follows on the generators:

$$
\left(\Lambda^{ \pm}\left(t_{l}^{k}\right)\right)_{n}^{m} \equiv\left(R_{ \pm}\right)_{l, n}^{k, m}, \quad\left(\Xi^{ \pm}\left(t_{l}^{k}\right)\right)_{n}^{m} \equiv\left(R^{ \pm}\right)_{l, n}^{k, m}, \quad\left(\Xi^{ \pm}(1)\right)_{n}^{m}=\left(\Lambda^{ \pm}(1)\right)_{n}^{m}=\delta_{n}^{m} .
$$

Here and in what follows, we set:

$$
R^{+} \equiv \mathbf{P} R \mathbf{P} ; \quad R^{-} \equiv R^{-1} ; \quad R_{+} \equiv R ; \quad R_{-} \equiv \mathbf{P} R^{-1} \mathbf{P},
$$

where $\mathbf{P} \in \operatorname{End}\left(\mathbf{C}^{N} \otimes \mathbf{C}^{N}\right)$ is defined as: $\mathbf{P}(x \otimes y) \equiv y \otimes x$. Moreover $\left(M_{N}(\mathbf{C})\right)^{\text {opp }}$ denotes the algebra $M_{N}(\mathbf{C})$ with the opposite multiplication.

We denote the entries of the above representations with the corresponding lower case greek letters $\left(\lambda^{ \pm}\right)_{j}^{i} ;\left(\xi^{ \pm}\right)_{j}^{i}$. Notice that the matrices $\Lambda^{ \pm}$and $\Xi^{ \pm}$, with entries in $U_{R}^{1}$, can be matrix-multiplied, but the entries of the products are not necessarily any more the entries of a representation of $A_{R}$ into $M_{N}(\mathbf{C})$ or $M_{N}(\mathbf{C})^{\text {opp }}$.

We have the following comultiplication rules:

$$
\nabla\left(\left(\lambda^{ \pm}\right)_{j}^{i}\right)=\sum_{s}\left(\lambda^{ \pm}\right)_{s}^{i} \otimes\left(\lambda^{ \pm}\right)_{j}^{s} ; \quad \nabla\left(\left(\xi^{ \pm}\right)_{j}^{i}\right)=\sum_{s}\left(\xi^{ \pm}\right)_{j}^{s} \otimes\left(\xi^{ \pm}\right)_{s}^{i}
$$

while the counit $\eta^{\prime}: U_{R}^{1} \rightarrow \mathbf{C}$ is given by $\eta^{\prime}(1)=1 ; \eta^{\prime}\left(\left(\lambda^{ \pm}\right)_{k}^{i}\right)=\eta^{\prime}\left(\left(\xi^{ \pm}\right)_{k}^{i}\right)=\delta_{k}^{i}$.

The following relations ${ }^{2}$ hold in $U_{R}^{1}$ :

$$
\begin{gathered}
\sum_{i, j}\left(R^{+}\right)_{i, j}^{k, l}\left(\lambda^{\varepsilon}\right)_{p}^{i}\left(\lambda^{\varepsilon}\right)_{m}^{j}=\sum_{i, j}\left(\lambda^{\varepsilon}\right)_{j}^{l}\left(\lambda^{\varepsilon}\right)_{i}^{k}\left(R^{+}\right)_{p, m}^{i, j}, \quad \varepsilon= \pm, \\
\sum_{i, j}\left(R^{+}\right)_{i, j}^{k, l}\left(\lambda^{+}\right)_{p}^{i}\left(\lambda^{-}\right)_{m}^{j}=\sum_{i, j}\left(\lambda^{-}\right)_{j}^{l}\left(\lambda^{+}\right)_{i}^{k}\left(R^{+}\right)_{p, m}^{i, j}, \\
\sum_{i, j}\left(R_{+}\right)_{i, j}^{k, l}\left(\xi^{\varepsilon}\right)_{p}^{i}\left(\xi^{\varepsilon}\right)_{m}^{j}=\sum_{i, j}\left(\xi^{\varepsilon}\right)_{j}^{l}\left(\xi^{\varepsilon}\right)_{i}^{k}\left(R_{+}\right)_{p, m}^{i, j}, \quad \varepsilon= \pm, \\
\sum_{i, j}\left(R_{+}\right)_{i, j}^{k, l}\left(\xi^{+}\right)_{p}^{i}\left(\xi^{-}\right)_{m}^{j}=\sum_{i, j}\left(\xi^{-}\right)_{j}^{l}\left(\xi^{+}\right)_{i}^{k}\left(R_{+}\right)_{p, m}^{i, j}, \\
\Xi^{ \pm} \Lambda^{\mp}=\Lambda^{ \pm} \Xi^{\mp}=1 .
\end{gathered}
$$

We now set $\left(R^{T_{2}}\right)_{k, l}^{i, j} \equiv R_{k, j}^{i, l}$ and assume that $R^{T_{2}}$ and $\left(R^{-1}\right)^{T_{2}}$ are invertible. Then there exists an invertible $\mu \in \operatorname{End}\left(\mathbf{C}^{N}\right)$ such that $\mu \otimes \mu$ commutes with $R$ and the

\footnotetext{
${ }^{1}$ As far as the coalgebra structure of $U_{R}$ is concerned, notice that, if we do not want to assume the existence of a completed tensor product $U_{R} \hat{\otimes} U_{R}$, then we can simply consider the comultiplication $\nabla$ as a map

$$
\nabla: \operatorname{Hom}\left(A_{R}, \mathbf{C}\right) \rightarrow \operatorname{Hom}\left(A_{R} \otimes A_{R}, \mathbf{C}\right)
$$

with some suitable properties obtained by rephrasing the usual properties of the comultiplication. This consideration is not needed for $U_{R}^{1}$, which is bialgebra in the ordinary sense

${ }^{2}$ As we shall see later on, there are in general other relations
} 
following equation is satisfied [5]:

$$
R^{T_{2}}(\operatorname{Id} \otimes \mu)\left(R^{-1}\right)^{T_{2}}\left(\operatorname{Id} \otimes \mu^{-1}\right)=1 \in \operatorname{End}\left(C^{N} \otimes C^{N}\right) .
$$

So $U_{R}^{1}$ becomes an Hopf algebra with the following antipode:

$$
\gamma\left(\Lambda^{ \pm}\right) \equiv \Xi^{\mp} ; \quad \gamma\left(\Xi^{ \pm}\right) \equiv \mu \Lambda^{\mp} \mu^{-1} .
$$

As a consequence we have the following other constraints on the generators of $U_{R}^{1}$ :

$$
\left[\mu \Lambda^{ \pm} \mu^{-1}\right]^{T}\left(\Xi^{\mp}\right)^{T}=\left(\Xi^{ \pm}\right)^{T}\left[\mu \Lambda^{\mp} \mu^{-1}\right]^{T}=1,
$$

where the symbol ${ }^{T}$ means "transposed" (remember that our matrices have entries in $U_{R}^{1}$ ).

We now consider four maps (see [6]) $\widehat{\mathscr{R}}_{ \pm}$and $\hat{\mathscr{R}}^{ \pm}$from $A_{R}$ to $U_{R}^{1}$, which are defined as follows on the generators

$$
\widehat{\mathscr{R}}_{ \pm}\left(t_{b}^{a}\right) \equiv\left(\lambda^{ \pm}\right)_{b}^{a}, \quad \hat{\mathscr{R}}^{ \pm}\left(t_{b}^{a}\right) \equiv\left(\xi^{ \pm}\right)_{b}^{a}, \quad \hat{\mathscr{R}}^{ \pm}(1)=\widehat{\mathscr{R}}_{ \pm}(1) \equiv 1
$$

and are extended respectively as algebra anti-homomorphisms $\left(\widehat{\mathscr{R}}_{ \pm}\right)$and as algebra homomorphisms $\left(\widehat{\mathscr{R}}^{ \pm}\right)$. Hence $\widehat{\mathscr{R}}_{ \pm}$is a coalgebra homomorphism, while $\hat{\mathscr{R}}^{ \pm}$is a coalgebra anti-homomorphism.

We define furthermore $\mathscr{R}_{ \pm}, \mathscr{R}^{ \pm} \in \operatorname{Hom}\left(A_{R} \otimes A_{R}, \mathbf{C}\right)$ as follows:

$$
\mathscr{R}_{ \pm}\left(t_{\alpha} \otimes t_{\beta}\right) \equiv\left(\hat{\mathscr{R}}_{ \pm}\left(t_{\beta}\right)\right)\left(t_{\alpha}\right), \quad \mathscr{R}^{ \pm}\left(t_{\alpha} \otimes t_{\beta}\right) \equiv\left(\widehat{\mathscr{R}}^{ \pm}\left(t_{\beta}\right)\right)\left(t_{\alpha}\right) ; \quad t_{\alpha}, t_{\beta} \in A_{R} .
$$

$\mathscr{R}_{+}$is (an implicit expression for) the universal $R$-matrix and will also be denoted by the simpler symbol $\mathscr{R}$.

One can prove the following theorems:

$$
\begin{gathered}
\mathscr{R}^{\mp} \mathscr{R}_{ \pm}=1 \in \operatorname{Hom}\left(A_{R} \otimes A_{R}, \mathbf{C}\right) ; \\
\mathscr{R}_{ \pm}\left(t_{1}, t_{2}\right)=\mathscr{R}^{ \pm}\left(t_{2}, t_{1}\right) \quad \forall t_{1}, t_{2} \in A_{R} ; \\
(\mathrm{Id} \otimes \nabla) \mathscr{R}_{ \pm}=\left(\mathscr{R}_{ \pm}\right)_{1,3}\left(\mathscr{R}_{ \pm}\right)_{1,2} ; \quad(\nabla \otimes \mathrm{Id}) \mathscr{R}_{ \pm} \doteq\left(\mathscr{R}_{ \pm}\right)_{1,3}\left(\mathscr{R}_{ \pm}\right)_{2,3} ; \\
(\mathrm{Id} \otimes \nabla) \mathscr{R}^{ \pm}=\left(\mathscr{R}^{ \pm}\right)_{1,2}\left(\mathscr{R}^{ \pm}\right)_{1,3} ; \quad(\nabla \otimes \mathrm{Id}) \mathscr{R}^{ \pm}=\left(\mathscr{R}^{ \pm}\right)_{2,3}\left(\mathscr{R}^{ \pm}\right)_{1,3} .
\end{gathered}
$$

Moreover for any $v \in U_{R}$ we have the quasi-cocommutativity property

$$
\nabla^{\prime}(v) \mathscr{R}=\mathscr{R} \nabla(v) ; \quad \nabla(v) \mathscr{R}^{+}=\mathscr{R}^{+} \nabla^{\prime}(v) ;
$$

where $\nabla^{\prime}$ is the opposite comultiplication. Finally $\mathscr{R}$ (as well as $\mathscr{R}_{-}$and $\mathscr{R}^{ \pm}$) satisfies the Yang-Baxter equation:

$$
\mathscr{R}_{1,2} \mathscr{R}_{1,3} \mathscr{R}_{2,3}=\mathscr{R}_{2,3} \mathscr{R}_{1,3} \mathscr{R}_{1,2} .
$$

It is now possible to prove that we can choose the matrix $\mu$ which appears in $(1.11)($ see $[7,5,8])$ so that for a suitable invertible $\alpha \in \operatorname{End}\left(\mathbf{C}^{N}\right)$ satisfying: $[R, 1 \otimes \alpha]=$ $[1 \otimes \alpha, R]=0$ we have:

$$
\begin{gathered}
m\left(\left(1 \otimes \mu^{-1}\right) \mathbf{P} R \mathbf{P}\right)=\alpha \quad \text { and } \quad m\left(\left(1 \otimes \mu^{-1}\right) R^{-1}\right)=\alpha^{-1}, \\
m\left((1 \otimes \mu) \mathbf{P} R^{-1} \mathbf{P}\right)=\alpha^{-1} \quad \text { and } \quad m((1 \otimes \mu) R)=\alpha .
\end{gathered}
$$

Here $m: \operatorname{End}\left(\mathbf{C}^{N} \otimes \mathbf{C}^{N}\right) \rightarrow \operatorname{End}\left(\mathbf{C}^{N}\right)$ is the multiplication.

We can now consider the representations: $V_{\mu}^{ \pm}: A_{R} \rightarrow \mathbf{C} 1 \subset M_{N}(\mathbf{C})$ defined on the generators as: $V_{\mu}^{ \pm}\left(t_{b}^{a}\right) \equiv\left(\mu^{ \pm 1}\right)_{b}^{a} 1$. The set of the entries of these representations consists of one group-like element $v_{\mu} \in U_{R}$ and its inverse $v_{\mu}^{-1}$. 
We can adjoin $v_{\mu}^{ \pm 1}$ to $U_{R}^{1}$ obtaining a larger Hopf algebra, say $U_{R}^{2}$. In order to do so it is enough to set $\gamma\left(v_{\mu}^{ \pm 1}\right) \equiv v_{\mu}^{\mp 1}$.

We now set:

$$
\psi_{\mu}^{ \pm} \equiv\left[\mathscr{R}_{ \pm}\left(\mathrm{Id} \otimes v_{\mu}^{-1}\right)\right] \Delta^{\prime}=\left[\mathscr{R}^{ \pm}\left(\mathrm{Id} \otimes v_{\mu}\right)\right] \Delta^{\prime},
$$

where $\Delta^{\prime}$ is the opposite of the comultiplication $\Delta$ on $A_{R}$. The element $\psi_{\mu}^{-}$is usually called the ribbon element ([5]); its inverse is $\psi_{\mu}^{+}$. Moreover one can prove that the comultiplication of $\psi_{\mu}^{ \pm}$is given by:

and that we have:

$$
\nabla\left(\psi_{\mu}^{ \pm}\right)=\left(\psi_{\mu}^{ \pm} \otimes \psi_{\mu}^{ \pm}\right) \mathscr{R}^{ \pm} \mathscr{R}_{ \pm}
$$

$$
\psi_{\mu}^{ \pm} v=v \psi_{\mu}^{ \pm} \quad \forall v \in U_{R} .
$$

In other words starting from an invertible Yang-Baxter matrix $R \in \operatorname{End}\left(\mathbf{C}^{N} \otimes \mathbf{C}^{N}\right)$, such that $R^{T_{2}}$ and $\left(R^{-1}\right)^{T_{2}}$ are invertible, we have constructed a Hopf algebra which is "essentially" quasitriangular and ribbon.

In order to have a quasitriangular ribbon Hopf algebra in the sense of [9] and of [10] we need to have an antipode on $A_{R}$ which is dual to the antipode $\gamma(1.12)$ and which moreover descends to $A_{R} / K_{R}$, where $K_{R} \subset A_{R}$ is the bi-ideal generated by the kernel of $U_{R}^{1}$.

This problem will be addressed in the next section, for the special case when $R \in \operatorname{End}\left(\mathbf{C}^{N} \otimes \mathbf{C}^{N}\right)$ is the Yang-Baxter matrix related to link-invariants for $\Sigma \times I$. In the last section of this paper an explicit presentation of the relevant quantum group, via generators and relations, will be provided.

A direct proof of all the statements contained in this introduction is in [11].

\section{Multi-Parameter Quantum Groups and Quantum Determinants}

In this section we want to apply the FRT construction to the Yang-Baxter matrix $R \in \operatorname{End}\left(\mathbf{C}^{N} \otimes \mathbf{C}^{N}\right)$ given by:

$$
R_{k, l}^{i, j}\left(x, z_{k, l}\right)= \begin{cases}x^{2} & \text { if } i=j=k=l \\ z_{k, l}^{2} & \text { if } i=k \neq l=j \\ x^{2}-x^{-2} & \text { if } i=l>k=j \\ 0 & \text { otherwise }\end{cases}
$$

where $x$ and $z_{k, l}$ are non-zero complex numbers with $z_{l, k}=z_{k, l}^{-1}$. When we set $z_{k, l}=1 \forall k, l$ we obtain the Yang-Baxter matrix relevant to the fundamental representation of $\mathscr{U}_{x}(s l(N))$. Such multi-parameter Yang-Baxter matrices are considered in [12].

On the other side the matrix $R\left(x, z_{k, l}\right)$ is obtained by "twisting" the matrix $R(x) \equiv R\left(x, z_{k, l}=1\right)$ with the following diagonal matrix $M \equiv M\left(z_{k, l}\right) \in \operatorname{End}\left(\mathbf{C}^{N} \otimes \mathbf{C}^{N}\right)$ :

$$
M_{k, l}^{i, j}\left(z_{k, l}\right)= \begin{cases}1 & \text { if } i=j=k=l \\ z_{k, l} & \text { if } i=k \neq l=j \\ 0 & \text { otherwise }\end{cases}
$$

$\left(z_{l, k}=z_{k, l}^{-1}\right)$ 
By "twisting" we mean that the following equation holds: $R\left(x, z_{k, l}\right)=$ $M\left(z_{k, l}\right) R(x) M\left(z_{k, l}\right)$. The matrix $M$ satisfies the Yang-Baxter equation and the equation: $\mathbf{P} M \mathbf{P}=M^{-1}$.

In a more general setting we have the following statement [12]:

2.1 Theorem. Let $R \in \operatorname{End}\left(\mathbf{C}^{N} \otimes \mathbf{C}^{N}\right)$ be a Yang-Baxter matrix and let $M$ defined as in (2.2). Then MRM is also a Yang-Baxter matrix. Moreover if $\mu \in \operatorname{End}\left(\mathbf{C}^{N}\right)$ is invertible, $\mu \otimes \mu$ commutes with $R$ and Eqs. (1.11), (1.22) and (1.23) are satisfied, then $\mu \otimes \mu$ commutes with MRM and (1.11),(1.22) and (1.23) are satisfied with $R$ replaced by MRM (without modifying $\alpha \in \operatorname{End}\left(\mathbf{C}^{N}\right)$ ).

In the case of (2.1) the matrix $\mu \in \operatorname{End}\left(\mathbf{C}^{N}\right)$ can be chosen as

$$
\mu=\operatorname{diag}\left(x^{2(N-1)}, x^{2(N-3)}, \ldots, x^{2(1-N)}\right),
$$

with $\alpha=x^{2 N} 1$. The Hecke relation for $R\left(x, z_{k, l}\right)(2.1)$ is the same for any value of the parameters $z_{k, l}$, namely we have:

$$
\mathbf{P} R\left(x, z_{k, l}\right)-R\left(x, z_{k, l}\right)^{-1} \mathbf{P}=\left(x^{2}-x^{-2}\right) \mathbf{I}
$$

From now on, we assume that the parameter $x$ satisfies the following condition:

$$
x^{4} \neq-1
$$

We can now consider the following two projection operators $[4]$ in $\operatorname{End}\left(\mathbf{C}^{N} \otimes \mathbf{C}^{N}\right)$ :

$$
P_{+} \equiv \frac{\mathbf{P} R+x^{-2}}{x^{2}+x^{-2}}, \quad P_{-} \equiv \frac{-\mathbf{P} R+x^{2}}{x^{2}+x^{-2}},
$$

where we set here and from now on, $R \equiv R\left(x, z_{k, l}\right)$.

The above projection operators are the quantum analogues of the symmetrization and, respectively, of the antisymmetrization operator; they satisfy the following relations:

$$
\mathbf{P} R=x^{2} P_{+}-x^{-2} P_{-} ; \quad P_{+}+P_{-}=\mathbf{I}, \quad P_{+} P_{-}=P_{-} P_{+}=0
$$

and also

$$
P_{ \pm}=M^{-1}\left(P_{ \pm}\right)_{z_{k, l}=1} M .
$$

Following Gurevich [13], we consider the quantum exterior algebra $\Lambda_{q}^{*}\left(\mathbf{C}^{N}\right)$ defined as the quotient of the tensor algebra of the vector space $\mathbf{C}^{N}$ with respect to the two-sided ideal generated by the image of $P_{+}$.

The quantum exterior algebra is a graded algebra: $\Lambda_{q}^{*}\left(\mathbf{C}^{N}\right)=\sum_{k} \Lambda_{q}^{k}\left(\mathbf{C}^{N}\right)$.

Analogously to the ordinary exterior algebra case we denote by the symbol $a \wedge_{q} b$ the image of $a \otimes b$ in $\Lambda_{q}\left(\mathbf{C}^{N}\right)$. We have now:

2.2 Theorem. $A$ basis of $\Lambda_{q}^{k}\left(\mathbf{C}^{N}\right)$ is given by $\left\{e_{i_{1}} \wedge_{q} e_{i_{2}} \wedge_{q} \cdots \wedge_{q} e_{i_{k}}\right\}$ with $i_{1}<i_{2}<\cdots<i_{k}$.

Proof. From the equations:

$$
\sum_{i, j} R_{k, l}^{i, j}\left(e_{j} \wedge_{q} e_{i}\right)+x^{-2}\left(e_{k} \wedge_{q} e_{l}\right)=0 \quad \forall k, l
$$


we have immediately:

$$
e_{l} \wedge_{q} e_{l}=0, \quad \forall l ; \quad e_{k} \wedge_{q} e_{l}=-x^{2} z_{k, l}^{2}\left(e_{l} \wedge_{q} e_{k}\right), \quad \forall k>l,
$$

and there are no further relations between the elements $\left\{e_{i} \wedge_{q} e_{j}\right\}$. Hence the quantum exterior algebra is not alternating and in particular given any $a \in \mathbf{C}^{N}, a \wedge_{q} a$ is not necessarily zero ${ }^{3}$.

We consider now the quantum analogue of the antisymmetric tensor; it is denoted by the symbol $u_{\sigma(1) \sigma(2) \cdots \sigma(N)}$ and, by definition, it satisfies the equation:

$$
e_{\sigma(1)} \wedge_{q} e_{\sigma(2)} \wedge_{q} \cdots \wedge_{q} e_{\sigma(N)}=u_{\sigma(1) \sigma(2) \cdots \sigma(N)} e_{1} \wedge_{q} e_{2} \wedge_{q} \cdots \wedge_{q} e_{N}
$$

for any permutation $\sigma$ of $1,2, \ldots, N$. It is given by the following expression:

$$
u_{\sigma(1) \sigma(2) \cdots \sigma(N)}=(-x)^{2 l(\sigma)} \prod_{\operatorname{trans}(\sigma)} z_{k, l}^{2},
$$

where $l(\sigma)$ is the length of the permutation $\sigma$ and the product is extended over all the transpositions $e_{k} \wedge_{q} e_{l} \rightarrow e_{l} \wedge_{q} e_{k}$ which are needed in order to transform $e_{\sigma(1)} \wedge_{q} e_{\sigma(2)} \wedge_{q} \cdots \wedge_{q} e_{\sigma(N)}$ into $e_{1} \wedge_{q} e_{2} \wedge_{q} \cdots \wedge_{q} e_{N}$, irrespective of the chosen sequence of transpositions ${ }^{4}$.

If we transpose the projection operators (2.5) we obtain the projection operators $P_{+}^{T}$ and $P_{-}^{T}$ which satisfy Eqs. (2.6) with $R$ replaced by $\mathbf{P} R^{T} \mathbf{P}$. We have a corresponding quantum exterior algebra $\left(\Lambda^{T}\right)_{q}^{*}\left(\mathbf{C}^{N}\right)$ with wedge product $\wedge_{q}^{T}$.

The relevant quantum antisymmetric tensor $v^{\sigma(1) \sigma(2) \cdots \sigma(N)}$ is defined by the equation:

$$
e_{\sigma(1)} \wedge_{q}^{T} e_{\sigma(2)} \wedge_{q}^{T} \cdots \wedge_{q}^{T} e_{\sigma(N)}=v^{\sigma(1) \sigma(2) \cdots \sigma(N)} e_{1} \wedge_{q}^{T} e_{2} \wedge_{q}^{T} \cdots \wedge_{q}^{T} e_{N}
$$

and it is given by:

$$
v^{\sigma(1) \sigma(2) \cdots \sigma(N)} \equiv(-x)^{2 l(\sigma)} \prod_{\operatorname{trans}(\sigma)} z_{k, l}^{-2}
$$

where the product is extended exactly as in (2.9).

The definition of both $u$ and $v$ is then extended to the case when we are given an arbitrary set of indices; we simply set both the above tensors to be equal to zero when two repeated indices appear. Moreover, given any permutation $\sigma$, we will use the shortened notation $u_{\sigma}$ and $v^{\sigma}$ instead of $u_{\sigma(1) \sigma(2) \cdots \sigma(N)}$ and $v^{\sigma(1) \sigma(2) \cdots \sigma(N)}$.

Notice that if $z_{k, l}=1, \forall k, l$, then we have $\mathbf{P} R^{T} \mathbf{P}=R, P_{ \pm}^{T}=P_{ \pm}$and $u=v$.

We now have the following:

2.3 Definition. The quantum determinant $D_{q}$ in $A_{R}$ is defined as:

$$
D_{q} \equiv \sum_{\sigma} u_{\sigma} t_{1}^{\sigma(1)} t_{2}^{\sigma(2)} \cdots t_{N}^{\sigma(N)} \text {. }
$$

\footnotetext{
${ }^{3}$ As a comparison, note that the quantum analogue of the symmetric algebra satisfies the following relations (the notation is evident):

$$
e_{k} \otimes_{s \mid q} e_{l}=x^{-2} z_{k, l}^{2}\left(e_{l} \otimes_{s \mid q} e_{k}\right) \quad \forall k>l,
$$

and so the quantum symmetric algebra is not commutative

${ }^{4}$ I.e. the minimum number of transpositions of contiguous vectors which are needed in order to transform $e_{\sigma(1)} \wedge_{q} e_{\sigma(2)} \wedge_{q} \cdots \wedge_{q} e_{\sigma(N)}$ into $e_{1} \wedge_{q} e_{2} \wedge_{q} \cdots \wedge_{q} e_{N}$
} 
By considering the basic relations in $A_{R}$ we can prove:

2.4 Theorem. For any set of indices $i_{1}, i_{2}, \ldots, i_{N}$, we have the following identities in $A_{R}$ :

$$
\begin{aligned}
& u_{i_{1} i_{2} \cdots i_{N}} D_{q}=\sum_{\sigma} u_{\sigma} t_{i_{1}}^{\sigma(1)} t_{i_{2}}^{\sigma(2)} \cdots t_{i_{N}}^{\sigma(N)} \\
& v^{i_{1} i_{2} \cdots i_{N}} D_{q}=\sum_{\sigma} v^{\sigma} t_{\sigma(1)}^{i_{1}} t_{\sigma(2)}^{i_{2}} \cdots t_{\sigma(N)}^{i_{N}}
\end{aligned}
$$

where the sums are extended over the set of all permutations $\sigma$ of $\{1,2, \ldots, N\}$.

2.5 Corollary. $D_{q}$ is group-like.

Proof. From (2.13) we have immediately:

$$
\Delta\left(D_{q}\right)=\sum_{\sigma, \rho} u_{\sigma} t_{\rho(1)}^{\sigma(1)} t_{\rho(2)}^{\sigma(2)} \cdots t_{\rho(N)}^{\sigma(N)} \otimes t_{1}^{\rho(1)} t_{2}^{\rho(2)} \cdots t_{N}^{\rho(N)}=D_{q} \otimes D_{q}
$$

2.6 Theorem. If $z_{k, l} \neq 1$, then $D_{q}$ is not a central element. Namely we have

$$
t_{k}^{l} D_{q}=\left(\prod_{s} z_{k, s}^{2} z_{s, l}^{2}\right) D_{q} t_{k}^{l} .
$$

We denote now by the symbol $A_{R}^{\#}$ the bialgebra obtained from the bialgebra $A_{R}$ by adjoining the group-like element $\left(D_{q}\right)^{-1}$. We want to prove that $A_{R}^{\#}$ is an Hopf algebra. In order to define an antipode in $A_{R}^{\#}$ we need to consider a quantum comatrix, namely a collection of $N^{2}$ elements of $A_{R}$ denoted by $\tilde{T}_{R}=\left\{\left(\tilde{t}_{R}\right)_{j}^{i}\right\}$ satisfying the equation:

$$
\sum_{j} t_{j}^{i}\left(\tilde{t}_{R}\right)_{k}^{j}=D_{q} \delta_{k}^{i}
$$

The previous equation defines in fact a right quantum comatrix. The corresponding left comatrix $\tilde{T}_{L}=\left\{\left(\tilde{t}_{L}\right)_{j}^{i}\right\}$ is, by definition, the collection of $N^{2}$ elements of $A_{R}$ satisfying the equation:

$$
\sum_{j}\left(\tilde{t}_{L}\right)_{j}^{i} t_{k}^{j}=D_{q} \delta_{k}^{i}
$$

2.7 Theorem. The right and left comatrices exist and are unique. They are given by the following expressions:

$$
\begin{aligned}
& \left(\tilde{t}_{R}\right)_{j}^{i}=(-x)^{2(i-j)} \prod_{k<j} z_{j, k}^{2} \prod_{k<i} z_{k, i}^{2}\left(D_{q}\right)_{i}^{j}, \\
& \left(\tilde{t}_{L}\right)_{j}^{i}=(-x)^{2(i-j)} \prod_{k>j} z_{k, j}^{2} \prod_{k>i} z_{i, k}^{2}\left(D_{q}\right)_{i}^{j},
\end{aligned}
$$

where in both the above expressions the symbol $\left(D_{q}\right)_{j}^{i}$ denotes the quantum determinant of the matrix obtained from the matrix $T$ by eliminating the $i^{\text {th }}$ row and the $j^{\text {th }}$ column.

Proof. We set:

$$
v^{i} \equiv v^{i, 1, \ldots, i-1, i+1, \ldots, N}=\prod_{k<i}\left(-x^{2} z_{k, i}^{2}\right) .
$$

For any permutation $\sigma$ satisfying the condition $\sigma(1)=j$, we have $v^{\sigma}=v^{j} v^{\sigma(2), \sigma(3), \ldots, \sigma(N)}$. 
Hence from (2.14) we have:

$$
D_{q}=\sum_{j}\left(v^{i}\right)^{-1} v^{j} t_{j}^{i}\left[\sum_{\sigma \text { s.t. } \sigma(1)=j} v^{\sigma(2), \sigma(3), \ldots, \sigma(N)} t_{\sigma(2)}^{1} t_{\sigma(3)}^{2} \cdots t_{\sigma(i)}^{i-1} t_{\sigma(i+1)}^{i+1} \cdots t_{\sigma(N)}^{N}\right],
$$

where the term in square parentheses is the quantum determinant of the matrix obtained by deleting the $i^{\text {th }}$ row and the $j^{\text {th }}$ column.

Also from (2.14) we have:

$$
0=\sum_{j} v^{j} t_{j}^{k}\left[\sum_{\sigma \text { s.t. } \sigma(1)=j} v^{\sigma(2), \sigma(3), \ldots, \sigma(N)} t_{\sigma(2)}^{1} t_{\sigma(3)}^{2} \cdots t_{\sigma(i)}^{i-1} t_{\sigma(i+1)}^{i+1} \cdots t_{\sigma(N)}^{N}\right] \text { for } k \neq i
$$

This proves (2.18).

In order to prove (2.19) we have to perform completely analogous calculations in which we first define:

$$
u_{i} \equiv u_{1,2, \ldots, i-1, i+1, \ldots, N, i}=\prod_{k>i}\left(-x^{2} z_{k, i}^{2}\right)
$$

thus obtaining:

$$
D_{q}=\sum_{j}\left(u_{i}\right)^{-1} u_{j}\left[\sum_{\sigma \text { s.t. } \sigma(N)=j} u_{\sigma(1), \sigma(2), \ldots, \sigma(N-1)} t_{1}^{\sigma(1)} t_{2}^{\sigma(2)} \cdots t_{i-1}^{\sigma(i-1)} t_{i+1}^{\sigma(i)} \cdots t_{N}^{\sigma(N-1)}\right] t_{i}^{j},
$$

where again the term in square parentheses is the quantum determinant of the matrix obtained by deleting the $j^{\text {th }}$ row and the $i^{\text {th }}$ column.

From (2.15) we deduce:

\subsection{Theorem}

$$
D_{q}\left(\tilde{t}_{R}\right)_{j}^{i}=\left(\tilde{t}_{L}\right)_{j}^{i} D_{q} .
$$

The previous theorems allow us to define an antipode $S$ in $A_{R}^{\#}$. In fact we can set

$$
S\left(t_{j}^{i}\right) \equiv\left(\tilde{t}_{R}\right)_{j}^{i}\left(D_{q}\right)^{-1}=\left(D_{q}\right)^{-1}\left(\tilde{t}_{L}\right)_{j}^{i}
$$

Equations (2.16) and (2.17) guarantee that (2.22) is consistent with the requirement for an antipode. In particular we have:

$$
\sum_{j} t_{j}^{i} S\left(t_{k}^{j}\right)=\sum_{j} S\left(t_{j}^{i}\right) t_{k}^{j}=\delta_{k}^{i}
$$

Also from (2.16) and (2.17) we deduce the following comultiplication rules:

$$
\Delta\left(\tilde{t}_{R}\right)_{j}^{i}=\sum_{s}\left(\tilde{t}_{R}\right)_{j}^{s} \otimes\left(\tilde{t}_{R}\right)_{s}^{i} ; \quad \Delta\left(\tilde{t}_{L}\right)_{j}^{i}=\sum_{s}\left(\tilde{t}_{L}\right)_{j}^{s} \otimes\left(\tilde{t}_{L}\right)_{s}^{i}
$$

Due to the identities: $\eta\left(D_{q}\right)=1 ; \eta\left(\left(\tilde{t}_{R}\right)_{j}^{i}\right)=\eta\left(\left(\tilde{t}_{L}\right)_{j}^{i}\right)=\delta_{j}^{i}$, we have to set:

$$
\eta\left[\left(D_{q}\right)^{-1}\right] \equiv 1 ; \quad S\left(\left(D_{q}\right)^{ \pm 1}\right)=\left(D_{q}\right)^{\mp 1} .
$$

We want now to compute $S\left(\left(\tilde{t}_{R}\right)_{j}^{i}\right)$. From the previous equations we have:

2.9 Theorem. The following equivalent equations hold:

$$
\begin{aligned}
& S\left(\tilde{T}_{R}\right)=\left(D_{q}\right)^{-1}\left(\mu^{-1} T \mu\right), \\
& S\left(\tilde{T}_{L}\right)=\left(\mu^{-1} T \mu\right)\left(D_{q}\right)^{-1},
\end{aligned}
$$




$$
\begin{gathered}
S^{2}(T)=\mu^{-1} T \mu, \\
S^{2}\left(\tilde{T}_{R}\right)=\mu^{-1} \tilde{T}_{R} \mu, \\
S^{2}\left(\tilde{T}_{L}\right)=\mu^{-1} \tilde{T}_{L} \mu .
\end{gathered}
$$

Proof. We prove (2.28) namely we consider $S^{2}\left(t_{j}^{i}\right)=D_{q} S\left(\left(\tilde{t}_{R}\right)_{j}^{i}\right)$.

It is given by:

$$
\sum_{\pi} t_{1}^{\pi(1)} \cdots t_{N}^{\pi(N)} u_{\pi} \sum_{\sigma \text { s.t. } \sigma(i)=j} S\left(t_{1}^{\sigma(1)} \cdots t_{i-1}^{\sigma(i-1)} t_{i+1}^{\sigma(i+1)} \cdots t_{N}^{\sigma(N)}\right)\left(v^{j}\right)^{-1} v^{i} u_{\sigma[i, j]},
$$

here we have used the symbol $\sigma[i, j]$ to denote the permutation of $\{1,2, \ldots, j-1$, $j+1, \ldots, N\}$ associated to each permutation $\sigma$ satisfying the requirement $\sigma(i)=j$.

We define now

$$
\tilde{u}_{i} \equiv u_{i, 1,2, \ldots, N}=\prod_{k<i}\left(-x^{2} z_{i, k}^{2}\right)
$$

Equation (2.31) is in turn equal to:

i.e.

$$
\begin{aligned}
& \sum_{\sigma \text { s.t. } \sigma(i)=j} \sum_{\pi} t_{j}^{\pi(1)} t_{\sigma(1)}^{\pi(2)} \cdots t_{\sigma(i-1)}^{\pi(i)} t_{\sigma(i+1)}^{\pi(i+1)} \cdots t_{\sigma(N)}^{\pi(N)}\left(v^{j}\right)^{-1}\left(\tilde{u}_{j}\right)^{-1} v^{i} u_{\pi} \\
& \cdot S\left(t_{N}^{\sigma(N)}\right) S\left(t_{N-1}^{\sigma(N-1)}\right) \cdots S\left(t_{i+1}^{\sigma(i+1)}\right) S\left(t_{i-1}^{\sigma(i-1)}\right) \cdots S\left(t_{N}^{\sigma(N)}\right) \\
& =\sum_{\pi} t_{j}^{\pi(1)} \delta_{N}^{\pi(N)} \cdots \delta_{i+1}^{\pi(i+1)} \delta_{i-1}^{\pi(i)} \cdots \delta_{1}^{\pi(2)}\left(v^{j}\right)^{-1}\left(\tilde{u}_{j}\right)^{-1} v^{i} u_{\pi} \\
& =t_{j}^{i}\left(v^{j}\right)^{-1}\left(\tilde{u}_{j}\right)^{-1} \tilde{u}_{i} v^{i}=x^{4(i-j)} t_{j}^{i}=t_{j}^{i} \mu_{i}^{-1} \mu_{j}
\end{aligned}
$$

$$
S^{2}(T)=\mu^{-1} T \mu
$$

In conclusion we can state that the bialgebra $A_{R}^{\#}$ is a (non-involutive) Hopf algebra with bijective antipode.

Now we want to define the action of $U_{R}^{1}$ on $\left(D_{q}\right)^{-1}$. In this way we will establish a natural pairing between the two Hopf Algebras $A_{R}^{\#}$ and $U_{R}^{1}$.

First notice that we have

$$
\left(\lambda^{ \pm}\right)_{j}^{i}\left(D_{q}\right)=\rho_{i}^{ \pm} \delta_{j}^{i} ; \quad\left(\xi^{ \pm}\right)_{j}^{i}\left(D_{q}\right)=\left(\rho_{i}^{\mp}\right)^{-1} \delta_{j}^{i},
$$

where $\rho_{i}^{ \pm} \equiv x^{ \pm 2} \prod_{j \neq i} z_{i, j}^{2}$.

This implies that we have to set:

$$
\left(\lambda^{ \pm}\right)_{j}^{i}\left(\left(D_{q}\right)^{-1}\right) \equiv\left(\rho_{i}^{ \pm}\right)^{-1} \delta_{j}^{i} ; \quad\left(\xi^{ \pm}\right)_{j}^{i}\left(\left(D_{q}\right)^{-1}\right) \equiv \rho_{i}^{\mp} \delta_{j}^{i}
$$

As the element $v_{\mu}$ is concerned, notice that the equation $v_{\mu}^{ \pm 1}\left(D_{q}\right)=\operatorname{det}^{ \pm 1}(\mu)=1$ leads us to set $v_{\mu}^{ \pm 1}\left(\left(D_{q}\right)^{-1}\right) \equiv 1$. It is immediate to prove the following theorems:

2.10 Theorem. Let $\gamma$ be the antipode in $U_{R}^{1}$ and let $S$ be antipode in $A_{R}^{\#}$ defined before. We have:

$$
[\gamma(v)](t)=v[S(t)], \quad \forall t \in A_{R}^{\#}, \quad \forall v \in U_{R}^{1} .
$$

2.11 Theorem. When $R$ is given by (2.1), then the matrices $\Lambda^{ \pm}$and $\Xi^{ \pm}$are triangular, namely for $i>j$ we have $\left(\lambda^{+}\right)_{j}^{i}=0$ and $\left(\xi^{-}\right)_{j}^{i}=0$, while for $i<j$ we have $\left(\lambda^{-}\right)_{j}^{i}=0$ and $\left(\xi^{+}\right)_{j}^{i}=0$. Finally all the elements $\left(\xi^{ \pm}\right)_{i}^{i}$ and $\left(\lambda^{ \pm}\right)_{i}^{i}$ are group-like, they commute with each other and $\left(\xi^{ \pm}\right)_{i}^{i}=\left[\left(\lambda^{\mp}\right)_{i}^{i}\right]^{-1}$. 
We consider now the two-sided ideal $K_{R} \subset A_{R}^{\#}$ given by the kernel of $U_{R}^{1}$. The antipode $S$ maps $K_{R}$ into itself; hence $S$ descends to $A_{R}^{\#} / K_{R}$ and the latter algebra is a Hopf algebra. Furthermore we have, as a consequence of the previous considerations, that the Hopf algebras $A_{R}^{\#} / K_{R}$ and $U_{R}^{1}$ are dually paired and that the pairing is non-singular.

Here we use the same definition considered by Majid [14], namely two Hopf Algebras $H_{1}$ and $H_{2}$ are said to be dually paired if there exists a bilinear form $\langle\cdot, \cdot\rangle$ such that the multiplication of $H_{1}\left(H_{2}\right)$ is adjoint to the comultiplication of $H_{2}\left(H_{1}\right)$, the two antipodes are adjoint to each other and $\langle 1, y\rangle=\eta_{2}(y) ;\langle x, 1\rangle=$ $\eta_{1}(x) ; \forall x \in H_{1} ; y \in H_{2}$, where $\eta_{i}$ denotes the counit in $H_{i}$.

Now we want to consider the morphisms $\hat{\mathscr{R}}_{ \pm}$and $\hat{\mathscr{R}}^{ \pm}(1.14)$. Due to the triangularity of the matrices $\Lambda^{ \pm}$and $\Xi^{ \pm}$we have:

$$
\widehat{\mathscr{R}}_{ \pm}\left(D_{q}\right)=\prod_{i=1}^{N}\left(\lambda^{ \pm}\right)_{i}^{i} ; \quad \widehat{\mathscr{R}}^{ \pm}\left(D_{q}\right)=\prod_{i=1}^{N}\left(\xi^{ \pm}\right)_{i}^{i} ;
$$

and so we can set:

$$
\widehat{\mathscr{R}}_{ \pm}\left(\left(D_{q}\right)^{-1}\right) \equiv \widehat{\mathscr{R}}^{\mp}\left(D_{q}\right) ; \quad \widehat{\mathscr{R}}^{ \pm}\left(\left(D_{q}\right)^{-1}\right) \equiv \widehat{\mathscr{R}}_{\mp}\left(D_{q}\right) .
$$

From the definition above we can straightforwardly extend the definition of $\mathscr{R}_{ \pm}, \mathscr{R}^{ \pm}$ and of $\psi_{\mu}^{ \pm}$so to include their action on elements of $A_{R}^{\#} \otimes A_{R}^{\#}$ and respectively of $A_{R}^{\#}$.

2.12 Theorem. The following identities hold:

$$
\hat{\mathscr{R}}_{\mp}=\hat{\mathscr{R}}^{ \pm} \circ S ; \quad \hat{\mathscr{R}}^{\mp}=\hat{\mathscr{R}}_{ \pm} \circ S^{-1} \text {. }
$$

The existence of a bijective antipode in $A_{R}^{\#}$ and the ensuing Eqs. (2.33) allow us to express the four homomorphisms $\hat{\mathscr{R}}_{ \pm}$and $\hat{\mathscr{R}}^{ \pm}$in terms only of $\hat{\mathscr{R}}_{+}$and $\hat{\mathscr{R}}_{-}$ (or in terms of $\hat{\mathscr{R}}_{+}$and $\hat{\mathscr{R}}^{+}$if one prefers).

From (2.33) and from the identity: $\gamma \circ \widehat{\mathscr{R}}_{ \pm}=\hat{\mathscr{R}}^{\mp}$ other useful identities follow. In particular we have:

$$
\begin{aligned}
\gamma \circ \widehat{\mathscr{R}}_{ \pm} & =\widehat{\mathscr{R}}_{ \pm} \circ S^{-1} ; \quad \gamma^{-1} \circ \widehat{\mathscr{R}}_{ \pm}=\hat{\mathscr{R}}_{ \pm} \circ S ; \\
\gamma \circ \widehat{\mathscr{R}}^{ \pm} & =\widehat{\mathscr{R}}^{ \pm} \circ S^{-1} ; \quad \gamma^{-1} \circ \widehat{\mathscr{R}}^{ \pm}=\hat{\mathscr{R}}^{ \pm} \circ S .
\end{aligned}
$$

As a consequence we recover the standard properties of the universal $R$-matrix:

and

$$
\mathscr{R}_{ \pm}\left(S\left(t_{1}\right), t_{2}\right)=\mathscr{R}^{\mp}\left(t_{1}, t_{2}\right) ; \quad \mathscr{R}^{ \pm}\left(t_{1}, S\left(t_{2}\right)\right)=\mathscr{R}_{\mp}\left(t_{1}, t_{2}\right) \quad \forall t_{1}, t_{2} \in A_{R}^{\#}
$$

$$
\mathscr{R}_{ \pm}\left(S\left(t_{1}\right), S\left(t_{2}\right)\right)=\mathscr{R}_{ \pm}\left(t_{1}, t_{2}\right) ; \quad \mathscr{R}^{ \pm}\left(S\left(t_{1}\right), S\left(t_{2}\right)\right)=\mathscr{R}^{ \pm}\left(t_{1}, t_{2}\right) \quad \forall t_{1}, t_{2} \in A_{R}^{\#}
$$

As far as the ribbon element is concerned, notice that by setting $u \equiv v_{\mu}^{-1} \psi_{\mu}^{-}$ we have, $\forall t \in A_{R}^{\#}$ :

$$
u(t)=\mathscr{R}_{-}\left(S^{2} \otimes \mathrm{Id}\right) \Delta(t)=\mathscr{R}_{+}(\mathrm{Id} \otimes S) \Delta^{\prime}(t) ; \quad u^{-1}(t)=\mathscr{R}_{+}\left(\mathrm{Id} \otimes S^{2}\right) \Delta^{\prime}(t),
$$

and moreover:

$$
\begin{aligned}
\psi_{\mu}^{ \pm}(S(t)) & =\left[\mathscr{R}_{ \pm}\left(\mathrm{Id} \otimes v_{\mu}^{-1}\right)\right] \Delta^{\prime}(S(t))=\left[\mathscr{R}_{ \pm}\left(\mathrm{Id} \otimes v_{\mu}^{-1}\right)\right](S \otimes S) \Delta(t) \\
& =\left[\left(\operatorname{Id} \otimes v_{\mu}\right) \mathscr{R}_{ \pm}\right] \Delta(t)=\psi_{\mu}^{ \pm}(t) .
\end{aligned}
$$

Given any Hopf Algebra $H$, we denote now as usual by the symbol $H^{\text {opp }}$ the Hopf algebra with opposite multiplication. 
Also we denote by the symbol $U_{R}^{+}$the Hopf subalgebra of $U_{R}^{1}$ generated by the entries of $\left\{\Lambda^{+}\right\}$and $\left\{\Xi^{-}\right\}$and by the symbol $U_{R}^{-}$the Hopf subalgebra generated by the entries of $\left\{\Lambda^{-}\right\}$and $\left\{\Xi^{+}\right\}$.

We consider the following two-sided ideals of $A_{R}^{\# \text { : }}$

$$
K_{R}^{+} \equiv \operatorname{Ker}\left(\hat{\mathscr{R}}_{+}\right) ; \quad K_{R}^{-} \equiv \operatorname{Ker}\left(\hat{\mathscr{R}}_{-}\right)=\operatorname{Ker}\left(\hat{\mathscr{R}}^{+}\right) .
$$

As a consequence of the previous identities we have the following Hopf Algebra isomorphisms:

$$
\widehat{\mathscr{R}}_{+}: A_{R}^{\#} / K_{R}^{+} \rightarrow\left(U_{R}^{+}\right)^{\mathrm{opp}} ; \quad \hat{\mathscr{R}}_{-}: A_{R}^{\#} / K_{R}^{-} \rightarrow\left(U_{R}^{-}\right)^{\mathrm{opp}} .
$$

For any $t \in A_{R}^{\#}$ we denote now by $[t]_{+}$and by $[t]_{-}$the corresponding equivalence classes in $A_{R}^{\#} / K_{R}^{+}$and, respectively $A_{R}^{\#} / K_{R}^{-}$. We define the bilinear form:

$$
\left\langle\left[t_{2}\right]_{-},\left[t_{1}\right]_{+}\right\rangle_{\mathscr{R}} \equiv \mathscr{R}_{+}\left(t_{2}, t_{1}\right)=\mathscr{R}^{+}\left(t_{1}, t_{2}\right)=\mathscr{R}_{-}\left(S\left(t_{1}\right), t_{2}\right) .
$$

Notice that $\langle\cdot, \cdot\rangle_{\mathscr{R}}$ is a non-singular bilinear form: $A_{R}^{\#} / K_{R}^{-} \times A_{R}^{\#} / K_{R}^{+} \rightarrow \mathrm{C}$ (and consequently a non-singular bilinear form: $\left.\left(U_{R}^{-}\right)^{\text {opp }} \times\left(U_{R}^{+}\right)^{\text {opp }} \rightarrow \mathbf{C}\right)$.

On the other hand we have $U_{R}^{+}\left(K_{R}^{-}\right)=U_{R}^{-}\left(K_{R}^{+}\right)=0$ and from the isomorphisms (2.38) it follows that the dual pairing of the Hopf Algebras $A_{R}^{\#}$ and $U_{R}^{1}$ gives rise to a non-singular dual pairing $\langle\cdot, \cdot\rangle$ between the Hopf algebras $U_{R}^{+}$and $\left(U_{R}^{-}\right)^{\text {opp }}$ and between the Hopf algebras $U_{R}^{-}$and $\left(U_{R}^{+}\right)^{\text {opp }}$.

In other words, if we consider the (restricted) duality induced by the pairing $\langle\cdot, \cdot\rangle$, we have: $\left(U_{R}^{-}\right)^{*} \equiv\left(U_{R}^{+}\right)^{\text {opp }} ;\left(U_{R}^{+}\right)^{*} \equiv\left(U_{R}^{-}\right)^{\text {opp }}$, and the factorization property of the Universal $R$-matrix. Namely $\mathscr{R}$ can be seen as an element of $U_{R}^{+} \hat{\otimes} U_{R}^{-}$ where $\hat{\otimes}$ denotes a completed tensor product (so that formal power series $^{5}$ of ordinary tensor products are included).

The Hopf algebras $U_{R}^{ \pm}$are the multi-parameter generalizations of the $\mathscr{U}_{x}\left(b_{ \pm}\right)$, where $b_{ \pm}$are the Borel subalgebras of $s l(N)$.

Once we are given linear bases in $U_{R}^{+}$and in $U_{R}^{-}$which are mutually dual under the pairing $\langle\cdot, \cdot\rangle$, then we can compute explicitly the universal $R$-matrix for the multi-parameter quantum groups. A P.B.W. theorem can be proved in this case by adapting the arguments of Rosso [15]. This explicit calculation will be given elsewhere. The step which will be considered in the next section will be to express the algebra $U_{R}^{1}$ in terms of generators and relations.

For the time being, taking into account the results obtained so far, we can state the following:

2.13 Theorem. The FRT construction applied to the matrix (2.1) provides a multiparameter family of quasi-triangular ribbon Hopf Algebras.

Let us now compare briefly the situation when the matrix $R(3.1)$ depends on generic parameters $x$ and $z_{k, l}$ vs. the situation when $x$ is generic and $z_{k, l}=1, \forall k, l$. In the second situation (i.e. in the ordinary one-parameter quantum group) we have:

$$
\left(\lambda^{ \pm}\right)_{i}^{i}=\left(\xi^{ \pm}\right)_{i}^{i} \quad \forall i \quad\left(z_{k, l}=1\right) .
$$

In the one-parameter case usually the matrix $R \in \operatorname{End}\left(\mathbf{C}^{N} \otimes \mathbf{C}^{N}\right)$ is normalized by a constant factor, i.e. one consider, instead of $R$ the matrix $\widetilde{R} \equiv x^{-2 / N} R[4]$ so

${ }^{5}$ The variable $h$ for this power series expansion is obtained by setting $x=\exp (-h / 2)$ 
that $\operatorname{det}(\tilde{R})=1$. Let us denote the corresponding generators in $U_{\tilde{R}}^{1}$ by the symbols $\tilde{\Lambda}^{ \pm}$and $\widetilde{\Xi}^{ \pm}$. As a consequence of the normalization we have the following constraint on the generators of $U_{\tilde{R}}^{1}$ :

$$
\prod_{i=1}^{N}\left(\tilde{\lambda}^{ \pm}\right)_{i}^{i}=1 \quad\left(z_{k, l}=1\right) .
$$

In the generic multi-parameter case Eq. (2.40) is not valid any more. Moreover if we multiply the matrix $R$ given by (2.1), by the same factor $x^{-2 / N}$ (so that again we have $\operatorname{det} R=1$ ) then we obtain a constraint different from (2.41), namely we obtain:

$$
\prod_{i=1}^{N}\left(\tilde{\lambda}^{+}\right)_{i}^{i}=\prod_{i=1}^{N}\left(\tilde{\lambda}^{-}\right)_{i}^{i} .
$$

Hence in the one-parameter case $U_{\tilde{R}}^{+}$and $U_{\tilde{\tilde{R}}}$ have in common the set of $N$ generators $\left(\tilde{\lambda}^{+}\right)_{i}^{i}=\left(\tilde{\xi}^{+}\right)_{i}^{i}$, subjected to the relation (2.41), and their inverses $\left(\widetilde{\xi}^{-}\right)_{i}^{i}=\left(\tilde{\lambda}^{-}\right)_{i}^{i}$, while in the generic multi-parameter case $U_{\tilde{\mathbf{R}}}^{+}$and $U_{\tilde{\mathbf{R}}}$ have in common only the element (2.42) and its inverse $\prod_{i=1}^{N}\left(\tilde{\xi}^{-}\right)_{i}^{i}=\prod_{i=1}\left(\tilde{\xi}^{+}\right)_{i}^{i}$. Since the element $(2.42)$ is given by $\hat{\mathscr{R}}\left(D_{q}\right)=\hat{\mathscr{R}}_{-}\left(D_{q}\right)$ (with $R$ replaced by $\tilde{R}$ ), we will refer to it as the dual quantum determinant and denote it by the symbol $\Delta_{q}$. Like $D_{q}, \Delta_{q}$ is not a central element in the generic multi-parameter case.

As far as the quantum double [16] of $U_{\tilde{R}}^{+}$is concerned, this will be isomorphic to some tensor product of the quantum group times the abelian algebra generated by the dual quantum determinant $\Delta_{q}$ and its inverse. Again here we have a difference with respect to the one-parameter case, namely the introduction of many parameters removes a "degeneracy" of the quantum double.

\section{Generators and Relations}

In this section the Yang baxter matrix $R$ will be always given by (2.1). In fact we will mainly consider the matrix $\widetilde{R}$ obtained by dividing $R$ by its determinant.

We will give now an explicit presentation of $U_{\tilde{R}}^{1}$ in terms of generators and relations. From this construction it will be apparent that, for generic values of the parameters:

a) the number of independent generators of $U_{\tilde{R}}^{1}$ is given by the same number of independent generators of $\mathscr{U}(s l(N))$ plus $N$. This extra number of generators is due to the fact that corresponding to each generator of the Cartan subalgebra of $s l(N)$ we have two generators in $U_{\tilde{R}}^{1}$ and moreover we have the dual quantum determinant;

b) the relations among generators correspond to a multi-parameter quantum version of the Serre relations. Moreover when $z_{k, l} \mapsto 1$ then $U_{\tilde{R}}^{1}$ becomes $\mathscr{U}_{x}(s l(N))$. For these reasons we replace, from now on, the symbol $U_{\tilde{R}}^{1}$ with the symbol $\mathscr{U}_{x, z_{k, l}}(s l(N))$.

The basic relations for the generators of $U_{R}^{1}$ are expressed by Theorem 2.11 and the following theorems: 
3.1 Theorem. For $j \geqq k \geqq i$ we have:

$$
\left(\lambda^{+}\right)_{j}^{i}=x^{2}\left(x^{2}-x^{-2}\right)^{-1}\left(\lambda^{+}\right)_{j}^{k}\left(\xi^{-}\right)_{k}^{k}\left(\lambda^{+}\right)_{k}^{i}-x^{-2}\left(x^{2}-x^{-2}\right)^{-1}\left(\lambda^{+}\right)_{k}^{i}\left(\xi^{-}\right)_{k}^{k}\left(\lambda^{+}\right)_{j}^{k} ;
$$

while for $i \geqq k \geqq j$ we have:

$$
\left(\lambda^{-}\right)_{j}^{i}=x^{2}\left(x^{2}-x^{-2}\right)^{-1}\left(\lambda^{-}\right)_{k}^{i}\left(\xi^{+}\right)_{k}^{k}\left(\lambda^{-}\right)_{j}^{k}-x^{-2}\left(x^{2}-x^{-2}\right)^{-1}\left(\lambda^{-}\right)_{j}^{k}\left(\xi^{+}\right)_{k}^{k}\left(\lambda^{-}\right)_{k}^{i} \text {. }
$$

3.2 Corollary. Each one of the generators $\left(\lambda^{+}\right)_{j}^{i}, i<j$ can be expressed in terms of the generators $\left(\lambda^{+}\right)_{i+1}^{i}$ and $\left(\xi^{-}\right)_{k}^{k}$ and similarly each one of the generators $\left(\lambda^{-}\right)_{j}^{i}, i>j$ can be expressed in terms of the generators $\left(\lambda^{-}\right)_{i}^{i+1}$ and $\left(\xi^{+}\right)_{k}^{k}$.

3.3 Theorem. Each one of the generators $\left(\xi^{-}\right)_{j}^{i}, i<j$ can be expressed in terms of the generators $\left(\xi^{-}\right)_{i+1}^{i}$ and $\left(\lambda^{+}\right)_{k}^{k}$ and similarly each one of the generators $\left(\xi^{+}\right)_{j}^{i}, i>j$ can be expressed in terms of the generators $\left(\xi^{+}\right)_{i}^{i+1}$ and $\left(\lambda^{-}\right)_{k}^{k}$.

3.4 Theorem. The generators $\left(\xi^{-}\right)_{i+1}^{i}$ and $\left(\xi^{+}\right)_{i}^{i+1}$ can be expressed in terms of the generators $\left(\xi^{-}\right)_{i}^{i},\left(\xi^{-}\right)_{i+1}^{i+1},\left(\lambda^{+}\right)_{i+1}^{i}$ and respectively $\left(\xi^{+}\right)_{i+1}^{i+1},\left(\xi^{+}\right)_{i}^{i},\left(\lambda^{-}\right)_{i}^{i+1}$.

Proof. Equation (2.24) and the triangularity imply:

$$
\left(\xi^{-}\right)_{i}^{i}\left(\lambda^{+}\right)_{i+1}^{i}+\left(\xi^{-}\right)_{i+1}^{i}\left(\lambda^{+}\right)_{i+1}^{i+1}=0
$$

and a similar equation for $\left(\xi^{+}\right)_{i}^{i+1}$.

We are now left only with the following independent generators: $\left(\lambda^{+}\right)_{i+1}^{i},\left(\lambda^{-}\right)_{i}^{i+1},\left(\lambda^{ \pm}\right)_{i}^{i}$ and the inverses of the latter ones $\left(\xi^{\mp}\right)_{i}^{i}$. In the one-parameter case one has $\left(\xi^{ \pm}\right)_{i}^{i}=\left(\lambda^{ \pm}\right)_{i}^{i}$, while in the generic multi-parameter case, this is not true any more and that is the main difference between the two cases.

Now we are in a position to construct explicitly generators and relations for $\mathscr{U}_{x, z k, l}(s l(N))$. From now on we consider the renormalized matrix $\widetilde{R}$.

In order to have a better picture of the relation between the generators of the multi-parameter quantum group and the corresponding one-parameter generators, expressed in the most common form, we find it convenient to consider the square roots of $\left(\tilde{\lambda}^{ \pm}\right)_{i}^{i}$ and of $\left(\tilde{\xi}^{ \pm}\right)_{i}^{i}$.

More specifically we set for any $t \in A_{R}^{\# \text { : }}$

that is:

$$
\sqrt{\left(\tilde{\lambda}^{ \pm}\right)_{i}^{i}}(t) \equiv \sqrt{\left(\tilde{\lambda}^{ \pm}\right)_{i}^{i}(t)}
$$

$$
\begin{aligned}
& \sqrt{\left(\tilde{\lambda}^{+}\right)_{i}^{i}}\left(t_{l}^{k}\right)=x^{-1 / N} \delta_{l}^{k} z_{k, i}(k \neq i) ; \quad \sqrt{\left(\tilde{\lambda}^{-}\right)_{i}^{i}}\left(t_{l}^{k}\right)=x^{1 / N} \delta_{l}^{k} z_{k, i}(k \neq i) ; \\
& \sqrt{\left(\tilde{\lambda}^{ \pm}\right)_{i}^{i}}\left(t_{j}^{i}\right)=\delta_{j}^{i} x^{ \pm(1-1 / N)} .
\end{aligned}
$$

A similar definition is given for $\sqrt{\left(\tilde{\xi}^{ \pm}\right)_{i}^{i}}$.

The introduction of such square roots does not create any serious problem, since they are group-like elements. In fact it enables us to express the element $v_{\mu}$ in terms of these square roots as follows:

$$
v_{\mu}=\prod_{i=1}^{i=N-1}\left[\sqrt{\left(\tilde{\lambda}^{+}\right)_{i}^{i}} \sqrt{\left(\tilde{\xi}^{+}\right)_{i}^{i}}\right]^{2 i-N-1} .
$$

We are now ready for a redefinition of the generators. We set:

$$
E_{i}^{+} \equiv x \frac{1}{\left(x^{2}-x^{-2}\right)} z_{i, i+1}\left(\tilde{\lambda}^{+}\right)_{i+1}^{i} \sqrt{\left(\tilde{\xi}^{-}\right)_{i}^{i}\left(\tilde{\xi}^{-}\right)_{i+1}^{i+1}},
$$




$$
\begin{gathered}
E_{i}^{-} \equiv-x^{-1} \frac{1}{\left(x^{2}-x^{-2}\right)} z_{i+1, i}\left(\tilde{\lambda}^{-}\right)_{i}^{i+1} \sqrt{\left(\tilde{\xi}^{+}\right)_{i+1}^{i+1}\left(\tilde{\xi}^{+}\right)_{i}^{i}}, \\
K_{i}^{ \pm}=\sqrt{\left(\tilde{\lambda}^{ \pm}\right)_{i}^{i}\left(\tilde{\xi}^{\mp}\right)_{i+1}^{i+1}}, \\
K=\prod_{i=1}^{N} \sqrt{\left(\tilde{\lambda}^{ \pm}\right)_{i}^{i} .}
\end{gathered}
$$

Hence the independent generators of $\mathscr{U}_{x, z_{k, l}}(\operatorname{sl}(N))$ are $^{6}$ :

$$
E_{i}^{ \pm} ; K_{i}^{ \pm} ;\left(K_{i}^{ \pm}\right)^{-1} ; K ; K^{-1} ; i=1, \ldots, N-1 .
$$

(In the one-parameter case we have $K_{i}^{ \pm}=\left(K_{i}^{\mp}\right)^{-1}$ and $K=1$.)

$E_{i}^{ \pm}$satisfy the following comultiplication rules:

$$
\nabla\left(E_{i}^{ \pm}\right)=E_{i}^{ \pm} \otimes\left(K_{i}^{ \pm}\right)^{\mp 1}+\left(K_{i}^{ \pm}\right)^{ \pm 1} \otimes E_{i}^{ \pm},
$$

the other generators being group-like. The commutation relations become:

$$
\begin{gathered}
{\left[E_{i}^{\varepsilon}, E_{j}^{\varepsilon}\right]=0 ; \quad|i-j| \geqq 2, \quad(\varepsilon= \pm) ; \quad\left[E_{i}^{+}, E_{j}^{-}\right]=0 \quad \forall i, j ;} \\
{\left[K_{i}^{\varepsilon}, K_{j}^{\varepsilon}\right]=0 \quad \forall i, j(\varepsilon= \pm) ; \quad\left[K_{i}^{+}, K_{j}^{-}\right]=\left[K_{i}^{ \pm}, K\right]=0, \quad \forall i, j ;} \\
{\left[E_{i}^{+}, E_{i}^{-}\right]=-\left(x^{2}-x^{-2}\right)^{-1}\left(\left(K_{i}^{+}\right)^{-1} K_{i}^{-}-K_{i}^{+}\left(K_{i}^{-}\right)^{-1}\right), \quad \forall i ;}
\end{gathered}
$$

and the "quantum Serre relations":

$$
\begin{aligned}
& E_{i}^{+}\left(E_{i \pm 1}^{+}\right)^{2}+\left(E_{i \pm 1}^{+}\right)^{2} E_{i}^{+}=\left(x^{2}+x^{-2}\right) E_{i \pm 1}^{+} E_{i}^{+} E_{i \pm 1}^{+}, \quad \forall i \\
& E_{i \pm 1}^{-}\left(E_{i}^{-}\right)^{2}+\left(E_{i}^{-}\right)^{2} E_{i \pm 1}^{-}=\left(x^{2}+x^{-2}\right) E_{i}^{-} E_{i \pm 1}^{-} E_{i}^{-}, \quad \forall i .
\end{aligned}
$$

Finally the commutation relations between the group-like generators and the nongroup-like ones read as follows:

$$
\begin{array}{ll}
E_{i}^{+} K_{i}^{+}=x^{2} K_{i}^{+} E_{i}^{+} ; \quad E_{i}^{+} K_{i}^{-}=x^{-2} K_{i}^{-} E_{i}^{+}, & \forall i ; \\
E_{i}^{-} K_{i}^{-}=x^{2} K_{i}^{-} E_{i}^{-} ; \quad E_{i}^{-} K_{i}^{+}=x^{-2} K_{i}^{+} E_{i}^{-}, & \forall i ; \\
E_{i}^{+} K_{i+1}^{+}=x^{-1} z_{i, i+1} z_{i+1, i+2} z_{i+2, i} K_{i+1}^{+} E_{i}^{+}, & \forall i ; \\
E_{i}^{+} K_{i-1}^{+}=x^{-1} z_{i-1, i+1} z_{i, i-1} z_{i+1, i} K_{i-1}^{+} E_{i}^{+}, & \forall i ; \\
E_{i}^{-} K_{i+1}^{-}=x^{-1} z_{i+1, i} z_{i+2, i+1} z_{i, i+2} K_{i+1}^{-} E_{i}^{-}, & \forall i ; \\
E_{i}^{-} K_{i-1}^{-}=x^{-1} z_{i+1, i-1} z_{i-1, i} z_{i, i+1} K_{i-1}^{-} E_{i}^{-}, & \forall i ; \\
E_{i}^{+} K_{i+1}^{-}=x z_{i, i+1} z_{i+1, i+2} z_{i+2, i} K_{i+1}^{-} E_{i}^{+}, & \forall i ; \\
E_{i}^{+} K_{i-1}^{-}=x z_{i-1, i+1} z_{i, i-1} z_{i+1, i} K_{i-1}^{-} E_{i}^{+}, & \forall i ; \\
E_{i}^{-} K_{i+1}^{+}=x z_{i+1, i} z_{i+2, i+1} z_{i, i+2} K_{i+1}^{+} E_{i}^{-}, & \forall i ; \\
E_{i}^{-} K_{i-1}^{+}=x z_{i+1, i-1} z_{i-1, i} z_{i, i+1} K_{i-1}^{+} E_{i}^{-}, & \forall i ; \\
E_{i}^{+} K=z_{i, i+1}^{2} \prod_{j \neq i, i+1}\left(z_{j, i+1} z_{i, j}\right) K E_{i}^{+}, & \forall i ;
\end{array}
$$

\footnotetext{
${ }^{6}$ With the above definition of the $E_{l}^{ \pm}$'s we have $E_{i}^{+}\left(t_{l}^{k}\right)=\delta_{i+1}^{k} \delta_{l}^{i}$ and $E_{i}^{-}\left(t_{l}^{k}\right)=\delta_{i}^{k} \delta_{l}^{i+1}$
} 


$$
\begin{array}{ll}
E_{i}^{+} K_{j}^{+}=z_{j, i+1} z_{i, j} z_{i+1, j+1} z_{j+1, i} K_{j}^{+} E_{i}^{+}, & |i-j| \geqq 2 ; \\
E_{i}^{-} K_{j}^{-}=z_{i+1, j} z_{j, i} z_{j+1, i+1} z_{i, j+1} K_{j}^{+} E_{i}^{+}, & |i-j| \geqq 2 ; \\
E_{i}^{+} K_{j}^{-}=z_{j, i+1} z_{i, j} z_{i+1, j+1} z_{j+1, i} K_{j}^{-} E_{j}^{+}, & |i-j| \geqq 2 ; \\
E_{i}^{-} K_{j}^{+}=z_{i+1, j} z_{j, i} z_{j+1, i+1} z_{i, j+1} K_{j}^{+} E_{i}^{-}, & |i-j| \geqq 2 .
\end{array}
$$

Notice that if we assume:

$$
z_{i, k}=z \text { for } i>k ; \text { and } z_{i, k}=z^{-1} \text { for } i<k \text {, }
$$

then all the coefficients in Eqs. (3.25), (3.26), (3.27), (3.28) become simply 1. This assumption corresponds to the case concerning the construction of invariants of links in $\Sigma \times[0,1]$ ( $\Sigma$ being a non-trivial 2-dimensional surface), as is shown in [2].

Acknowledgements. We thank very much N. Reshetikhin for having explained to us some relevant facts concerning the construction of the ribbon element. We also would like to thank very much for discussions D. Barnes, R. Heyneman, S. Mathur, A. Mewborne, J. Stasheff. P.C.R. wants especially to thank J. Stasheff and the Mathematics Department of the University of North Carolina for very nice hospitality, during the preparation of this work.

\section{References}

1. Turaev, V. G.: Skein quantization of poisson algebras of loops on surfaces. LOMI preprint (July 1989)

2. Cotta-Ramusino, P., Rinaldi, M.: On the Algebraic structure of link-diagrams on a 2-dimensional surface. Commun. Math. Phys. 138, 137-173 (1991)

3. Cotta-Ramusino, P., Rinaldi, M.: Link Diagrams, Yang-Baxter equation and Quantum Holonomy. Report at the Amherst Conference on Quantum Groups and Deformations of Algebras (June 1990), submitted to Contemporary Mathematics

4. Reshetikhin, N. Yu., Takhadzhyan, L. A., Faddeev, L. D.: Quantization of Lie groups and Lie algebras. Leningrad Math. J. 1, 193-225 (1990)

5. Reshetikhin, N. Yu.: Quasitriangle Hopf algebras and invariants of tangles. Leningrad Math. J. 1, 491-513 (1990)

6. Majid, S.: Quasitriangular Hopf algebras and Yang-Baxter equations. Int. J. Mod. Phys. A, 5, 1-91 (1990)

7. Turaev, V. G.: The Yang-Baxter equation and invariants of links. Invent. Math. 92, 527-553 (1988)

8. Rinaldi, M.: Ph. Thesis, S.I.S.S.A., Trieste

9. Drinfel'd, V. G.: On almost cocommutative Hopf Algebras. Leningrad Math. J. 1, 321-342 (1990)

10. Reshetikhin, N. Yu., Turaev, V. G.: Invariants of 3-Manifolds via Link Polynomials and Quantum Groups. Invent. Math. 103, 547-597 (1991)

11. Cotta-Ramusino, P., Rinaldi, M.: Construction of quantum groups with many parameters. Preprint Harvard (March 1991)

12. Reshetikhin, N. Yu.: Multiparameter quantum groups and Twisted Quasitriangular Hopf Algebras. Lett. Math. Phys. 20, 331-335 (1990)

13. Gurevich, D. I.: Hecke symmetries and quantum determinants. Sov. Math. Dokl. 38, 555-559 (1989)

14. Majid, S.: More examples of bicrossproduct and double cross product Hopf Algebras. Israel J. Math. (1991)

15. Rosso, M.: An Analogue of P.B.W. theorem and the Universal $R$-Matrix for $U_{h} s l(n+1)$. Commun. Math. Phys. 124, 307-318 (1989)

16. Reshetikhin, N. Yu., Semenov-Tjian-Shansky, M. A.: Quantum R-Matrices and Factorization problems in Quantum Groups. J. Geom. Phys. 5, 533-550 (1988)

Communicated by N. Yu. Reshetikhin 